\title{
Quantitative evaluation methods for water- flooded layers of conglomerate reservoir based on well logging data
}

\author{
Tan Fengqii, ${ }^{1,}$, Li Hongqi ${ }^{1,2 *}$, Xu Changfu ${ }^{3}$, Li Qingyuan $^{3}$ and \\ Peng Shouchang ${ }^{3}$ \\ ${ }^{1}$ College of Geophysics and Information, China University of Petroleum, Beijing 102249, China \\ ${ }^{2}$ State Key Laboratory of Petroleum Resource and Prospecting, China University of Petroleum, Beijing 102249, China \\ ${ }^{3}$ Research Institute of Petroleum Exploration and Development, Xinjiang Oilfield Company, PetroChina, Karamay, Xinjiang \\ 834000 , China
}

(C) China University of Petroleum (Beijing) and Springer-Verlag Berlin Heidelberg 2010

\begin{abstract}
The rapid changing near source, multi-stream depositional environment of conglomerate reservoirs leads to severe heterogeneity, complex lithology and physical properties, and large changes of oil layer resistivity. Quantitative evaluation of water-flooded layers has become an important but difficult focus for secondary development of oilfields. In this paper, based on the analysis of current problems in quantitative evaluation of water-flooded layers, the Kexia Group conglomerate reservoir of the Sixth District in the Karamay Oilfield was studied. Eight types of conglomerate reservoir lithology were identified effectively by a data mining method combined with the data from sealed coring wells, and then a multi-parameter model for quantitative evaluation of the water-flooded layers of the main oil-bearing lithology was developed. Water production rate, oil saturation and oil productivity index were selected as the characteristic parameters for quantitative evaluation of water-flooded layers of conglomerate reservoirs. Finally, quantitative evaluation criteria and identification rules for water-flooded layers of main oil-bearing lithology formed by integration of the three characteristic parameters of water-flooded layer and undisturbed formation resistivity. This method has been used in evaluation of the water-flooded layers of a conglomerate reservoir in the Karamay Oilfield and achieved good results, improving the interpretation accuracy and compliance rate. It will provide technical support for avoiding perforation of high water-bearing layers and for adjustment of developmental programs.
\end{abstract}

Key words: Water-flooded layer, quantitative evaluation, conglomerate reservoir, lithology identification, decision tree, characteristic parameters

\section{Introduction}

The Kexia Group reservoir of the Sixth District in the Karamay Oilfield is located in the Baijiantan Area of the Xinjiang Uyghur Autonomous Region, NW China. It is cut into sealed triangle-like fault-blocks by surrounding faults. On the north is the Baijiantan fault, on the south is the Ke$\mathrm{Wu}$ fault and the bottom structure is an anticline retained by faults. The sedimentary environment of the Kexia Group reservoir is a set of positive cycle piedmont alluvial fans. The reservoir overlaps above the Carboniferous formation with a sedimentary thickness of 45-77 m. The sedimentary microfacies developed in the Kexia Group reservoir, include the main trough, lateral edge trough, trough beach

*Corresponding author. email: hq.li@cup.edu.cn

Received July 9, 2009 and sheetflood belt of top fan, the braided stream, braided sand island and overflow belt of middle fan, and the branch channel, point bar and marsh of edge fan. This corresponds to a typical conglomerate reservoir.

At present, the conglomerate reservoirs of the Karamay Oilfield are at the high water cut stage after 40 years of waterflood development. Especially for the main adjustment blocks of the old area, accurate evaluation of water-flooded layers at the high water cut phase has became a technical key for controlling water production and steadying oil production as well as increasing reserves and potentially improving the oil production in secondary development. However, because of the characteristics of the conglomerate reservoirs such as severe heterogeneity, complex lithology, double-model distribution of the micro-porous structure of a conglomerate reservoir as well as abnormal logging response, there is not yet effective identification method for quantitative evaluation 
of water-flooded layers, and the main reasons of which are as follows (Shen et al, 1998; Jiang et al, 2005): 1) It is very difficult to accurately identify the lithology. Because the special sedimentary environment of a conglomerate reservoir leads to complex and changeable reservoir lithology, it is difficult for conventional geophysics statistical methods to integrate various well logging information to extract lithology-sensitive parameters and establish an identification model, and the well logging response of different lithologies varies largely, especially in the resistivity curve. Hence, accurate identification of the lithology of a conglomerate reservoir is the basis for accurate calculation of oil saturation and quantitative evaluation of water-flooded layers; 2) It is difficult to establish a model for calculation of oil saturation. Because of the complex porous structure and severe heterogeneity of a conglomerate reservoir, the litho-electrical parameters vary over a wide range, hence it is very difficult to achieve satisfying accuracy when calculating oil saturation for a conglomerate reservoir based on the Achier equation and other corresponding deformation models. Establishing a model with variable parameters that can continuously reflect the change of formation information is essential for accurate calculation of oil saturation; 3) The sensitive parameters for water-flooded layers of a conglomerate reservoir need to be re-selected. Two characteristic parameters of oil saturation $\left(S_{\mathrm{o}}\right)$ and water production rate $\left(F_{\mathrm{w}}\right)$ are well applicable in evaluation of water-flooded layers of sandstone reservoirs with a single lithology and uniform properties, but for conglomerate reservoirs the sensitivity of these parameters are not adequate. Constructing a parameter which can eliminate the influence of reservoir framework and only reflect the change of nature of pore fluid is the key to evaluation of water-flooded layers; 4) At present, no evaluation criterion and identification rules for water-flooded layers of conglomerate reservoir have been reported either at home or abroad. In this paper, based on the actual geological features of a conglomerate reservoir, combined with well logging curves, and data from sealed coring wells and the dynamic development information of the conglomerate reservoir, the above four difficulties were studied and an effective method for quantitative evaluation of water-flooded layers of a conglomerate reservoir was obtained.

\section{Identification of lithology of conglomerate reservoir}

\subsection{Data mining methods}

The Kexia Group conglomerate reservoir is divided into two sand sections denoted as $\mathrm{S}_{6}$ and $\mathrm{S}_{7}$, and the lithology changes from shaly and sandy inequigranular conglomerate to conglomeratic inequigranular sand of middle fan, finally to conglomerate and shaly fine-silt sandstone of edge fan and flood plain, from the bottom to the top, so the lithology of the conglomerate reservoir is complex and changeable, and its oil-bearing properties change greatly (Fig. 1). With traditional mathematical statistics methods, it is difficult to accurately reflect nonlinear mapping relationships of well logging data and conglomerate lithology, but the data mining method with the ability of self-organization, self-learning, reasoning and thinking, and high-precision nonlinear modeling can give a good solution to this problem.

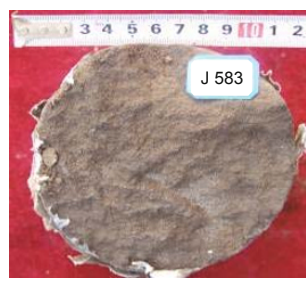

(1)

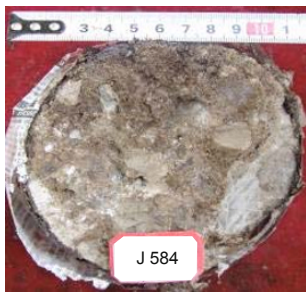

(6)

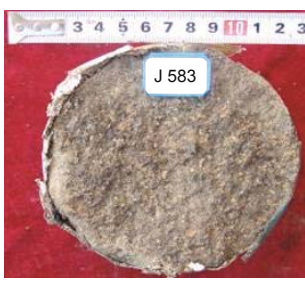

(2)

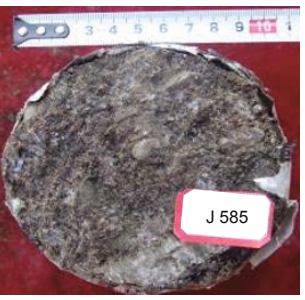

(7)

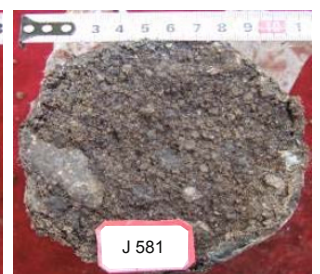

(3)

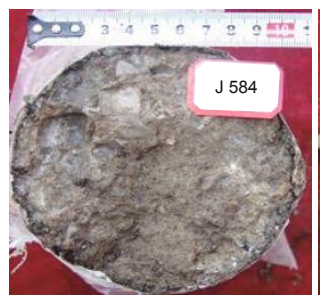

(8)

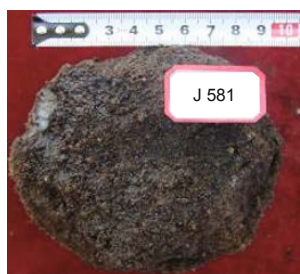

(4)

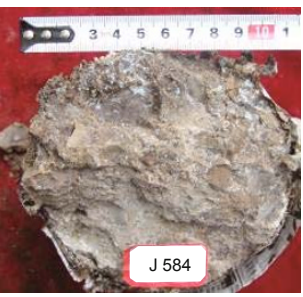

(9)

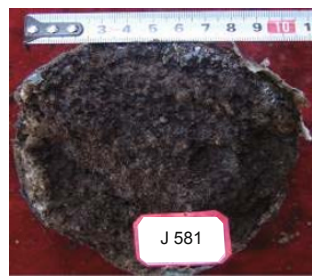

(5)

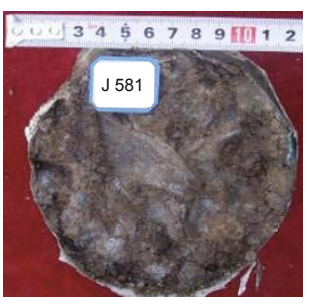

(10)

(1) Gray mudstone, medium; (2) Shaly conglomeratic sandstone, medium, oil spot; (3) Conglomeratic gritstone, medium, oil spot;

(4) Conglomeratic gritstone, medium, oil immersion; (5) Conglomeratic gritstone, loose, oil rich; (6) Glutenite, medium, oil spot;

(7) Glutenite, loose, oil spot; (8) Sandy conglomerate, tight, oil trace; (9) Sandy conglomerate, medium, oil spot;

(10) sandy conglomerate, medium, oil immersion

Fig. 1 Change of lithology and oil-bearing property of the Kexia Group conglomerate reservoir 
Data mining is a process of extracting potentially useful, hidden, unknown in advance, information and knowledge from mass, incomplete, noisy, fuzzy and random data (Fayyad, 1996). The tasks of data mining are classified into description and prediction. Description is making a summary through deduction of the potential relationship in data. Prediction is making forecasts through deduction of the current data. The modeling methods for forecasting come from machine learning, artificial intelligence, pattern identification, statistics and other research areas (Witten and Frank, 2005; Han and Kamber, 2006), and the main modeling methods include Neural Network, Support Vector Machines, Bayesian Network, and Decision Tree methods. The Decision Tree method is selected for the data mining of conglomerate lithology, based on the following two considerations. The first is that the model is established by the Decision Tree method is easy-to-read and easy-to-understand. The second is that the Decision Tree method is a "white-box" model, from which one can know how the classifier works and the importance of various parameters, supporting a guide for geophysics research.

The Decision Tree is a tree structure which is similar to flow chart. It starts to test the data samples from the root node and the data samples are divided into different subsets according to different results, and each subset of data samples forms a minor node (Tian, 2004). Each branch represents a test output, and each leaf node represents a category or category distribution. The basic algorithm of the Decision Tree is a greedy algorithm, which builds a decision tree by recursion from the top to the bottom, including two processes of construction and classification. The data mining process is a continuous cycle of optimization, and the data mining of the Decision Tree consists of six steps of data preprocessing, data selection, data transformation, data mining, model evaluation and knowledge discovery.

\subsection{Data mining of conglomerate lithology based on the Decision Tree method}

Identification of lithology by use of well logging data builds a correspondence relationship between well logging response characteristics and lithology. At present, the lithology of core samples of sandstone and volcanic rock has been accurately identified according to the cast thin slice, but for conglomerate reservoir, because of its severe heterogeneity and complex lithology, the cast thin slice of partial core can not accurately reflect the actual lithology of formation. Therefore, the data of sealed coring wells are used to study the lithology identification of the Kexia Group conglomerate reservoir. The lithological profile of the intervals from the sealed coring wells are built on the basis of sequence stratigraphy, and then the well logging lithology of the corresponding intervals of core description. Finally, the corresponding relationships between conglomerate lithology and well logging data of 117 intervals were established.

According to the grain-size the Kexia Group conglomerate lithology can be divided into eight types of lithology, namely conglomerate, sandy conglomerate, glutenite, conglomeratic gritstone, fine sandstone, conglomeratic mudstone, silty mudstone and mudstone. Seven parameters of undisturbed formation resistivity, natural gamma ray, spontaneous potential, well caliper, neutron porosity, acoustic travel time and compensated density logging are selected as the mining field to indicate lithology, and the mining results are shown in Fig. 2. As seen in Fig. 2, the Decision Tree method selects three sensitive parameters of resistivity, natural gamma gay and acoustic travel time from seven lithological characteristic parameters according to their weight on lithology to identify the lithology in four steps from the top to the bottom. Each branch of the tree represents a class of lithology identification rules and the leaf nodes express the attributive parameters of these identification rules as well as their numerical interval.

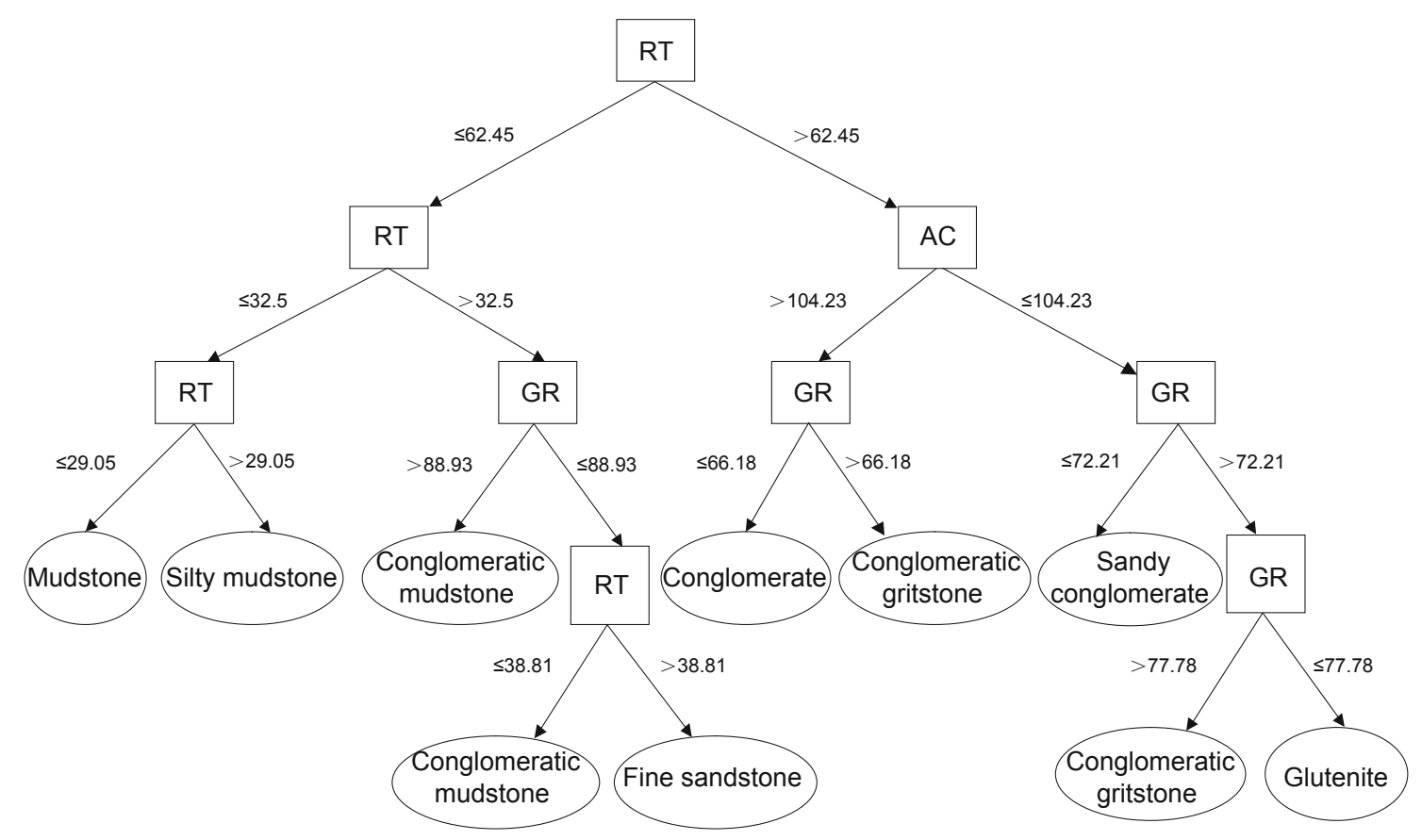

Fig. 2 The tree map of identification of conglomerate lithology based on the Decision Tree method 
Analysis of the model can clearly know how a lithology is being identified based on parameter association and correlative rules, and the identification accuracy can achieve $97.0 \%$.

\section{Establishment of a model for oil saturation of conglomerate reservoirs}

In view of the characteristics of conglomerate reservoirs, i.e. complex lithology and severe heterogeneity, and based on detailed analysis of the meaning of the parameters and their determination in various models for calculation of water saturation (such as the Archie equation, revised Archie equation, Simandoux equation and multiple linear regression equation) (Sun et al, 2008; Shen et al, 2008), and using the coring data of sealed coring wells, the parameters value for model calculation of characteristic conglomerate reservoir are eventually determined. The accuracy of various models in calculation of the water saturation of a conglomerate reservoir was analyzed (see Fig. 3) and a multiple linear regression equation was selected as the model for calculation of water saturation of the Kexia Group conglomerate reservoir. Because all the previous models for water saturation of reservoir are related to the formation water resistivity, and the influencing factors on the resistivity are large in number and complex, it is very difficult for these models to be accurately calculated. The multiple linear regression model uses the normalized value of continuous spontaneous potential curve to replace the formation water resistivity, improving the calculation accuracy of the saturation model. Equation (1) is used for calculation of the water saturation of a conglomeratic gritstone reservoir.

$$
\begin{aligned}
& \lg S_{\mathrm{w}}=-0.1985 \lg R_{t}-0.7674 \lg \phi+0.0283 \Delta S P+2.9836 \\
& R^{2}=0.847
\end{aligned}
$$

where $S_{\mathrm{w}}$ is the formation water saturation, $\% ; R_{t}$ is the undisturbed formation resistivity, $\Omega \cdot \mathrm{m}$; $\square$ is the effective porosity, $\% ; \triangle S P$ is the normalized value of spontaneous potential; $R$ is the correlation coefficient.

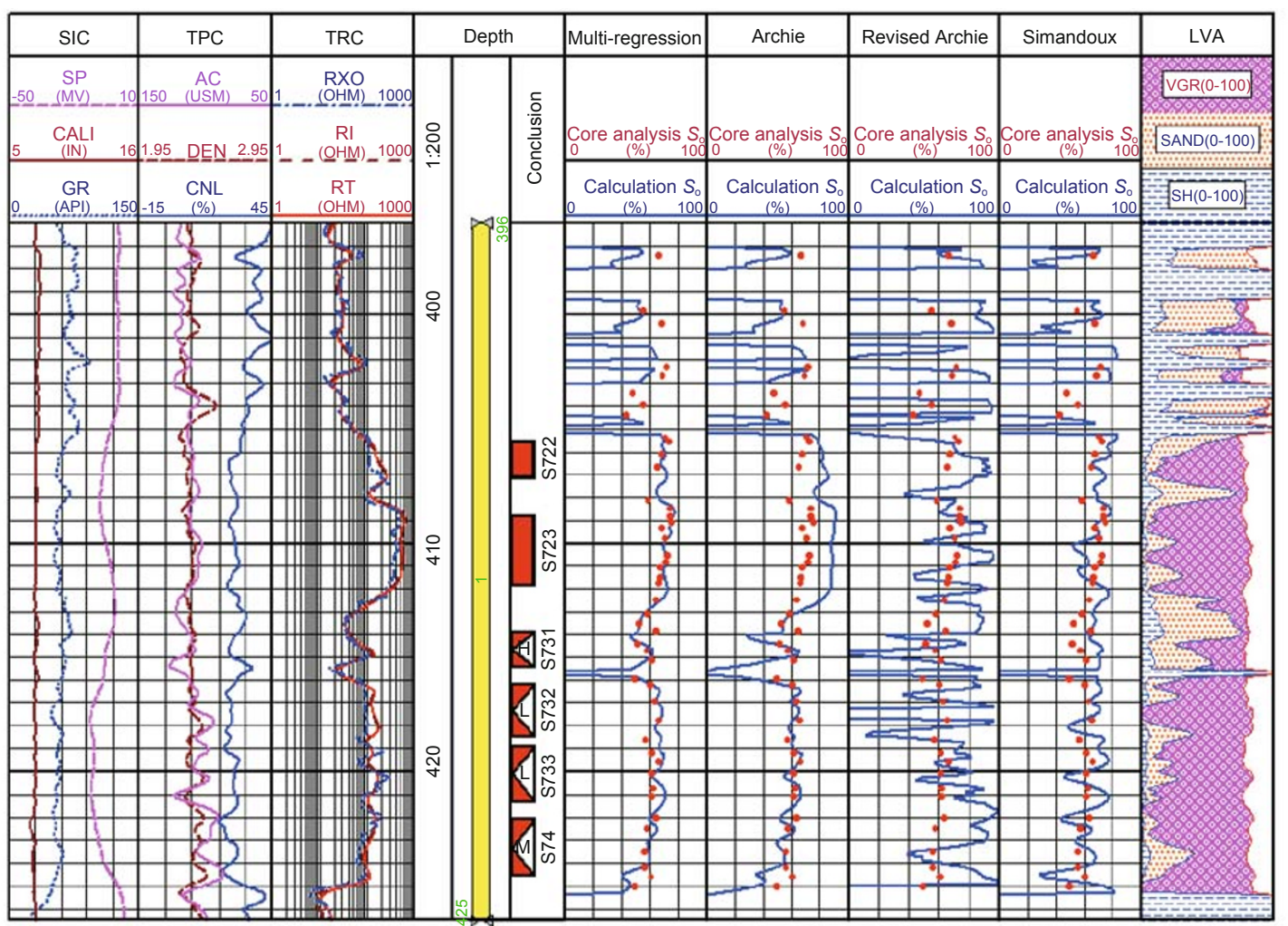

Notes: the red points are the oil saturation value of corrected core analysis and blue curves are results of model calculation.

Fig. 3 Comparison of calculated results of different saturation models

The lithology of the main oil-bearing reservoir of the Kexia Group conglomerate reservoir includes conglomeratic gritstone, glutenite and conglomerate, so the water saturation models were established according to different lithologies, and different models have different coefficients (see Table 1). In Fig. 3 the results of oil saturation for different models were calculated based on the correlation parameters of different lithologies and it can seen that the multiple linear regression equation and the Archie equation are the best in view of stability and accuracy of calculation, but for the Archie equation the formation water resistivity is a fixed value, so the sensitivity of this model is low and its accuracy is not as high as the multiple linear regression equation. The revised Archie equation needs to calculate the formation skeleton resistivity, which is attributed to the additional conductivity of clay minerals in the formation skeleton, and the formation water 
resistivity is calculated by use of the formation model of the flushed zone, in addition, because of the poor lithology and physical properties of the conglomerate reservoir, especially its severe heterogeneity, the results of the calculation of oil saturation are instable and very changeable not reflecting the actual oil saturation of the reservoir. For the Simandoux equation, the formation model is established based on sand and shale profile, not considering the impact of gravel content on the water saturation, therefore, this model is not suitable for a conglomerate reservoir.

Table 1 Parameters of saturation models of main oil-bearing lithology

\begin{tabular}{|c|c|c|c|c|c|c|}
\hline \multirow{2}{*}{$\begin{array}{c}\text { Models } \\
\text { Parameters }\end{array}$} & \multicolumn{4}{|c|}{ Water saturation models } & \multicolumn{2}{|c|}{$\begin{array}{c}\text { Initial oil } \\
\text { saturation models }\end{array}$} \\
\hline & $\lg R_{\mathrm{t}}, \Omega \cdot \mathrm{m}$ & $\lg \square, \%$ & $\triangle S P$ & Constant & $\lg (K / \square)$ & Constant \\
\hline $\begin{array}{c}\text { Conglomeratic } \\
\text { gritstone }\end{array}$ & $\square 0.1975$ & $\square 0.7674$ & 0.0283 & 2.9836 & $\square 8.054$ & 80.788 \\
\hline Glutenite & $\square 0.1998$ & $\square 0.7862$ & 0.1328 & 2.9986 & $\square 12.324$ & 79.874 \\
\hline Conglomerate & $\square 0.2219$ & $\square 0.1992$ & 0.0287 & 2.2516 & $\square 15.492$ & 76.836 \\
\hline
\end{tabular}

\section{Construction of sensitive parameters for water-flooded layer}

\subsection{Definition of the oil productivity index}

To construct the sensitivity parameters for waterflooded layers of a conglomerate reservoir, the influence of lithology and heterogeneity must be eliminated. Based on this consideration, the oil productivity index is defined as follows.

$$
F_{\text {ow }}=\frac{S_{\mathrm{o}}^{\prime}-S_{\mathrm{o}}}{S_{\mathrm{o}}^{\prime}}
$$

where $F_{\text {ow }}$ is the oil productivity index; $S_{\mathrm{o}}^{\prime}$ is the initial oil saturation, $\% ; S_{\mathrm{o}}$ is the current oil saturation, $\%$.

The oil productivity index represents a dynamic parameter of water-flooded oil layers, and it can reflect the water-flooded extent of the current reservoir. The oil productivity index eliminates the influence of conglomerate lithology by use of the relative value of oil saturation and avoids the shortcoming in quantitative evaluation of water-flooded extent by only using the current oil saturation.

\subsection{Calculation method for initial oil saturation}

The initial oil saturation of reservoir is defined as the percentage of the volume of crude oil to the volume of effective pores in original reservoir state (Pan and Wang, 2000), and three methods are usually used for determining the initial oil saturation, namely the method of direct measurement of core (Ouyang, 1994), the indirect method for determining (Worthington, 1996) and the calculation method with the capillary pressure curve (Yu, 1995). The initial oil saturation of the Kexia Group conglomerate reservoir determined by direct measurement of core is rarely reported. The indirect method for determining needs to establish a conductive model for the oil-gas reservoir based on the lithology and physical properties of the reservoir and the response characteristics of well logging curves to determine the initial oil saturation. However, because the lithology and physical properties of the conglomerate reservoir are complex and the response data of well logging is random, the calculation accuracy by the conductive model is low, hence the indirect method is not suitable to calculation of initial oil saturation for a conglomerate reservoir. The calculation method by using the capillary pressure curve has achieved very good results for sandstone reservoirs with a single lithology and good physical properties, but for conglomerate reservoir with complex lithology and oil-bearing properties, this method is not suitable for calculation of initial oil saturation because it can not accurately scale a standard ordinate transformation template of the capillary pressure curve.

Through the above analysis it can be seen that the effect of lithology must be eliminated for accurate calculation of initial oil saturation of conglomerate reservoir, and the best way to eliminate the effect of lithology is to establish a platform with the same lithology and investigate the relationship between physical properties and oil-bearing property of reservoir based on this platform. The Karamay Group of the Sixth District is divided into the Kexia Group and the Keshang Group, and the oil saturation of the Keshang Group can reflect the initial oil saturation of reservoir because its developmental extent is relatively low without large-scale water injection. The lithoelectrical parameters and mercury injection curves of the Kexia Group and the Keshang Group were compared based on the core analysis data, and the results showed that for the oil-bearing reservoirs with same lithology and similar porosity, the difference of $a, b, m$, or $n$ value ( $a$ and $m$ are cementation factor and cementation exponent of rock respectively and reflect the pore structure and cementing strength of rock matrix; $b$ and $n$ are saturation factor and saturation exponent respectively and reflect the change of resistivity index with increasing of water saturation) is relatively small and their mercury injection curves also remain consistent, indicating that the pore structure and the pore-throat distribution are similar for the same lithology reservoirs of the Kexia Group and the Keshang Group. Therefore, the oil saturation equation of the Keshang Group can be used to calculate the initial oil saturation of the Kexia Group reservoir.

The migration and accumulation of oil and gas is a very complex process (Wang and Zhao, 2002; He et al, 2006). Only when the following factors such as source rock, reservoir, cap rock, trap, migration and conservation were reasonably aligned in time and space can the hydrocarbon reservoir be finally formed (Zhang et al, 2008; Zhang et al, 2007; Fu et al, 2009). When oil and gas migrated into the reservoir, the pore spaces with high porosity and good permeability are occupied first and with increasing filling pressure and deepening oildrive-water process, oil and gas can gradually migrate into the pore spaces with low porosity and poor permeability and high capillary pressure. Therefore, the initial oil saturation of the reservoir is closely related to the reservoir quality factor $(\sqrt{K / \phi})$. The relationship between the initial oil saturation and reservoir quality factor was established from the data 
from sealed coring wells, and the parameters of the initial oil saturation model of different lithology reservoirs are shown in Table 1. Equation (3) is the calculation model for initial oil saturation of conglomeratic gritstone.

$$
\begin{aligned}
& S_{\mathrm{o}}^{\prime}=-8.054 \lg (K / \phi)+80.788 \\
& R^{2}=0.8084
\end{aligned}
$$

where, $S_{\mathrm{o}}^{\prime}$ is the initial oil saturation, $\% ; K$ is the reservoir permeability, $10^{-3} \mu \mathrm{m}^{2}$; $\square$ is the effective porosity, $\%$; $R$ is the correlation coefficient.

\section{Quantitative evaluation criteria and identification rules for water-flooded layers}

\subsection{Evaluation criteria of characteristic parameters of water-flooded layers}

Quantitative evaluation of water-flooded layers of conglomerate reservoirs is difficult because of their unique characteristics. In order to make use of the information of various parameters to comprehensively identify water-flooded levels and to improve the evaluation accuracy for waterflooded layers of conglomerate reservoir, three characteristic parameters of water-flooded layers (oil saturation, water production rate and oil productivity index) are selected to quantitatively evaluate water-flooded levels, and the determination of criteria of water-flooded levels for the three parameters are also based on the lithology identification. Fig. 4 is the crossplot of oil-water relative permeability, water saturation and water production rate of conglomeratic gritstone of sealed coring well and Fig. 5 is the crossplot of the oil productivity index and water production rate. The evaluation criteria of oil saturation and oil productivity index for different lithologies were respectively determined based on the criteria of five water-flooded levels of water production rate in Table 2.

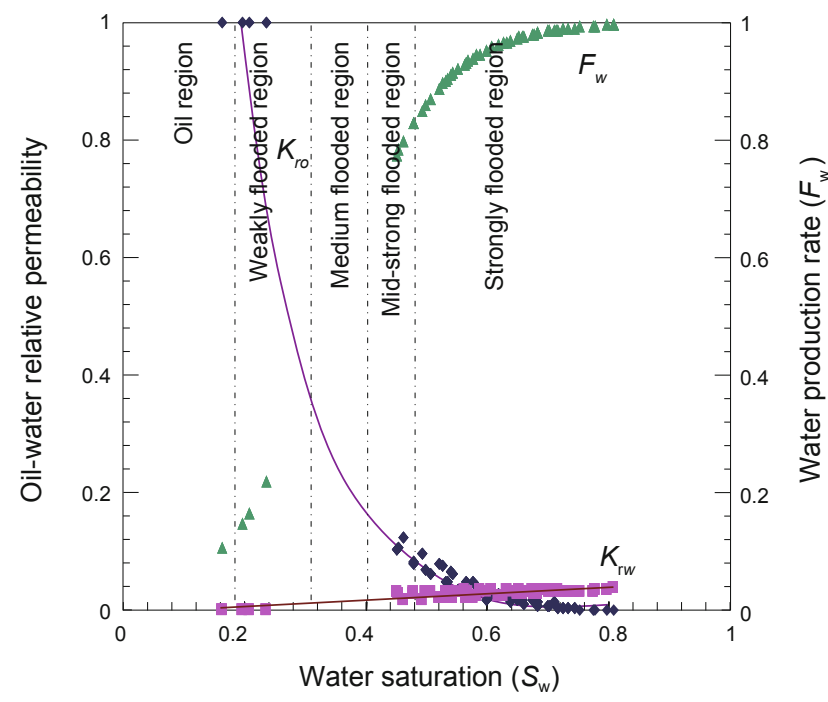

Fig. 4 The crossplot of water saturation, oil-water relative permeability and water production rate of conglomeratic gritstone

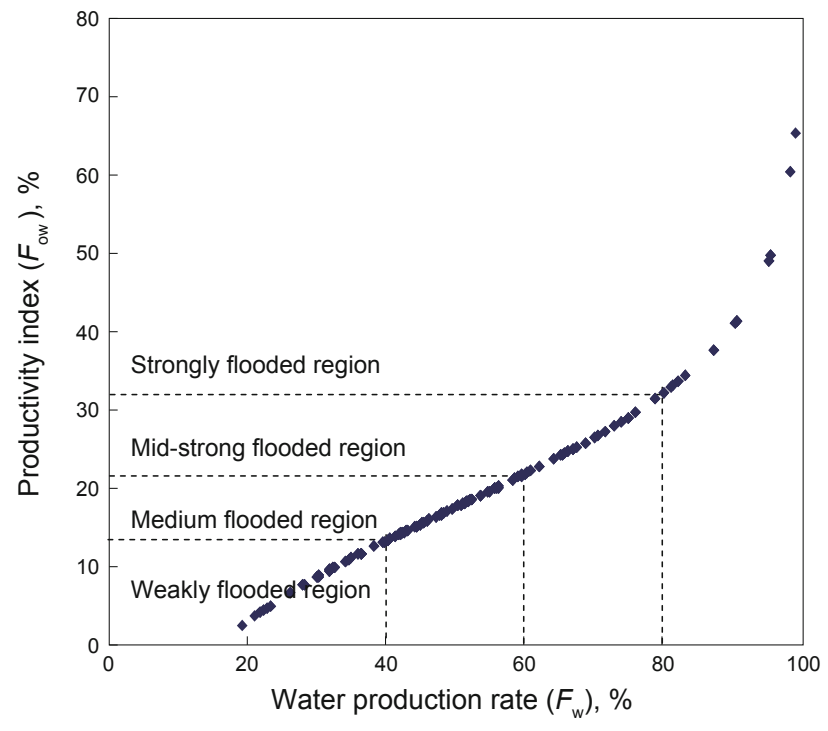

Fig. 5 The crossplot of oil productivity index and water production rate

\subsection{Evaluation criteria of reservoir resistivity}

The resistivity is a most sensitive parameter which can reflect the change of fluid properties in reservoir pores. In order to determine the relationship between resistivity and water-flooded levels of different lithologies, Through Casing Resistivity Logging was used to establish the evaluation criteria for them. The largest advantage of Through Casing Resistivity Logging is that it can measure the reservoir resistivity in the same lithology platform (Cheng and Ren, 2008), eliminating the influence of formation skeleton, clay minerals and conductive minerals, and that the variation of reservoir resistivity only reflects the change of pore fluid property, so Through Casing Resistivity Logging is the best in application and the evaluation accuracy of it is also the highest for conglomerate reservoirs with complex lithology and severe heterogeneity. Fig. 6 is the crossplot of reservoir resistivity and water production rate of conglomeratic gritstone, made by the measurement results of different periods by Through Casing Resistivity Logging. The evaluation criteria of water-flooded levels of reservoir resistivity for the main oil-bearing lithology of a conglomerate reservoir can be determined according to the identification criterion of water-flooded levels corresponding to water production rate (Table 2).

From Fig. 6 it can be seen that the reservoir resistivity gradually decreases with increasing water production rate and the decrease of reservoir resistivity is a two-slope process. The change of oil layer resistivity is different with the waterflooded extent for the two processes, so a point of inflexion appears on the curve of reservoir resistivity and the value of the reservoir resistivity at this inflexion point is usually defined as the inflexion saturation $\left(S_{\mathrm{wp}}\right)$. Generally the interconnected pores and throats in rocks form an integrated flow network, and in this flow system some pore fluid can not flow, some pore fluid can restrictively flow and some pore fluid can preferentially flow because of the influence of micro-heterogeneity in the water-drive-oil process, and 
Table 2 The quantitative evaluation criteria of water-flooded levels of different lithologies for conglomerate reservoir of Kexia Group in the Sixth District

\begin{tabular}{|c|c|c|c|c|c|c|}
\hline \multirow{2}{*}{ Block } & \multirow{2}{*}{ Lithology } & Water-flooded & \multicolumn{3}{|c|}{ Water production rate Oil saturation $\left(S_{\mathrm{o}}\right)$, Oil productivity index } & \multirow{2}{*}{$\begin{array}{l}\text { Reservoir resistivity } \\
\qquad\left(R_{\mathrm{t}}\right), \Omega \cdot \mathrm{m}\end{array}$} \\
\hline & & level & $\left(F_{\mathrm{w}}\right), \%$ & $\%$ & $\left(F_{\text {ow }}\right), \%$ & \\
\hline \multirow{15}{*}{$\begin{array}{l}\text { The Sixth } \\
\text { District }\end{array}$} & \multirow{5}{*}{$\begin{array}{c}\text { Conglomeratic } \\
\text { gritstone }\end{array}$} & Oil layer & $\leq 10$ & $\geq 82$ & $\leq 4$ & $\geq 170$ \\
\hline & & Weakly flooded layer & $10-40$ & $82-68$ & $4-14$ & $170-100$ \\
\hline & & Medium flooded layer & $40-60$ & $68-60$ & $14-22$ & $100-60$ \\
\hline & & Mid-strong flooded layer & $60-80$ & $60-50$ & $22-32$ & $60-45$ \\
\hline & & Strongly flooded layer & $\geq 80$ & $\leq 50$ & $\geq 32$ & $\leq 45$ \\
\hline & \multirow{5}{*}{ Glutenite } & Oil layer & $\leq 10$ & $\geq 78$ & $\leq 4$ & $\geq 200$ \\
\hline & & Weakly flooded layer & $10-40$ & $78-68$ & $4-12$ & $200-130$ \\
\hline & & Medium flooded layer & $40-60$ & $68-56$ & $12-20$ & $130-80$ \\
\hline & & Mid-strong flooded layer & $60-80$ & $56-47$ & $20-30$ & $80-60$ \\
\hline & & Strongly flooded layer & $\geq 80$ & $\leq 47$ & $\geq 30$ & $\leq 60$ \\
\hline & \multirow{5}{*}{ Conglomerate } & Oil layer & $\leq 10$ & $\geq 72$ & $\leq 3$ & $\geq 180$ \\
\hline & & Weakly flooded layer & $10-40$ & $72-60$ & $3-12$ & $180-110$ \\
\hline & & Medium flooded layer & $40-60$ & $60-52$ & $12-18$ & $110-70$ \\
\hline & & Mid-strong flooded layer & $60-80$ & $52-42$ & $18-26$ & $70-50$ \\
\hline & & Strongly flooded layer & $\geq 80$ & $\leq 42$ & $\geq 26$ & $\leq 50$ \\
\hline
\end{tabular}

these large pores with preferential flow form the main flow network. In the water-drive-oil process the crude oil of the major flow network is displaced first, and the water in the major flow network becomes the continuous phase and greatly influences the electrical property of rocks when the water-drive-oil process reaches certain extent. $S_{\mathrm{wp}}$ represents the water saturation when the injected water forms the major flow network, but not the maximum value of water saturation in water-drive-oil process.

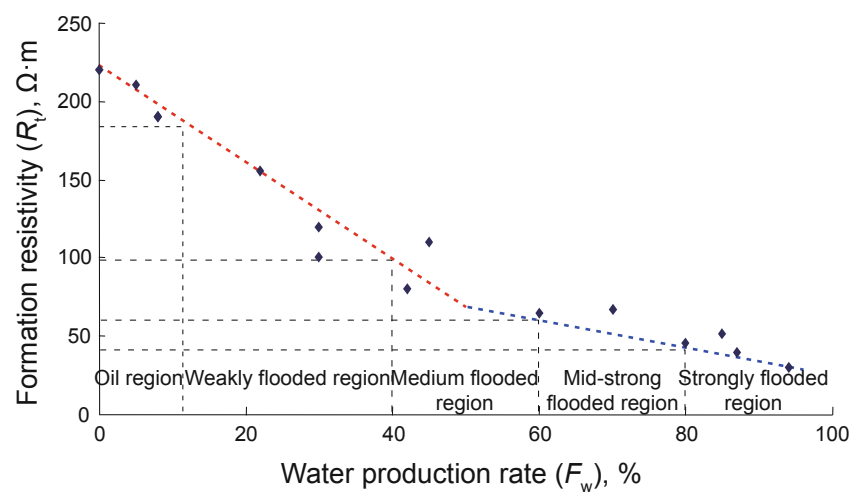

Fig. 6 The crossplot of reservoir resistivity and water production rate of conglomeratic gritstone

\subsection{Rules for evaluation of water-flooded layers of a conglomerate reservoir}

The rules of quantitative identification of water flooded layers in the Kexia Group conglomerate reservoir are mainly established by using three characteristic parameters of water- flooded layers, and assisted by using reservoir resistivity. The specific method are as follows.

(1) If the calculated results of the three characteristic parameters of water-flooded layers are all in the same range of water-flooded level, this oil layer is considered to be of the water-flooded level of this range.

(2) If the calculated results of two characteristic parameters of water-flooded layers among the three parameters are in the same range of water-flooded level and the third is not in this range, the oil layer is considered to be of the water-flooded level of this range.

(3) If the calculated results of the three parameters are different with each other, the water-flooded level of the oil layer is determined by use of reservoir resistivity and one of the three characteristic parameters of water-flooded layer based on the criteria for evaluation of water-flooded level by using reservoir resistivity of different lithologies.

\section{Application of this method and its effect}

The evaluation method and identification rules were applied to quantitative evaluation of water-flooded layers for the Kexia Group conglomerate reservoir, and achieved very good results, and the comprehensive interpretation results well agreed with actual production (see Fig. 7).

The interpretation of water-flooded layers for a conglomerate reservoir is a comprehensive evaluation process, including the study of the relationship between water injection and liquid production, the analysis of injection-production channels and water injection profile, the identification of water-flooded characteristics based on well 
logging curves, and qualitative interpretation and quantitative evaluation of water-flooded layers. The water-flooded layers of conglomerate reservoir in the Sixth District were evaluated according to the above evaluation process combined with specific identification methods. Fig. 7 shows the results of quantitative interpretation of water-flooded layers for well J581, which is an important sealed coring well of the Sixth District in 2008. In Fig. 7, the red curve shows the current oil saturation and blue curve shows the initial oil saturation of the conglomerate reservoir, and the difference between them shows the volume of crude oil being displaced in the reservoir after water-flood, and the ratio of it to initial oil saturation is the oil productivity index, indicating the waterflooded level of oil layers from the beginning of waterflood to the current state of the reservoir. Based on the three characteristic parameters of water-flooded layers and the reservoir resistivity the water-flooded levels of conglomerate reservoir were interpreted by use of the identification rules for water-flooded layers.

The Kexia Group is divided into five sand sections

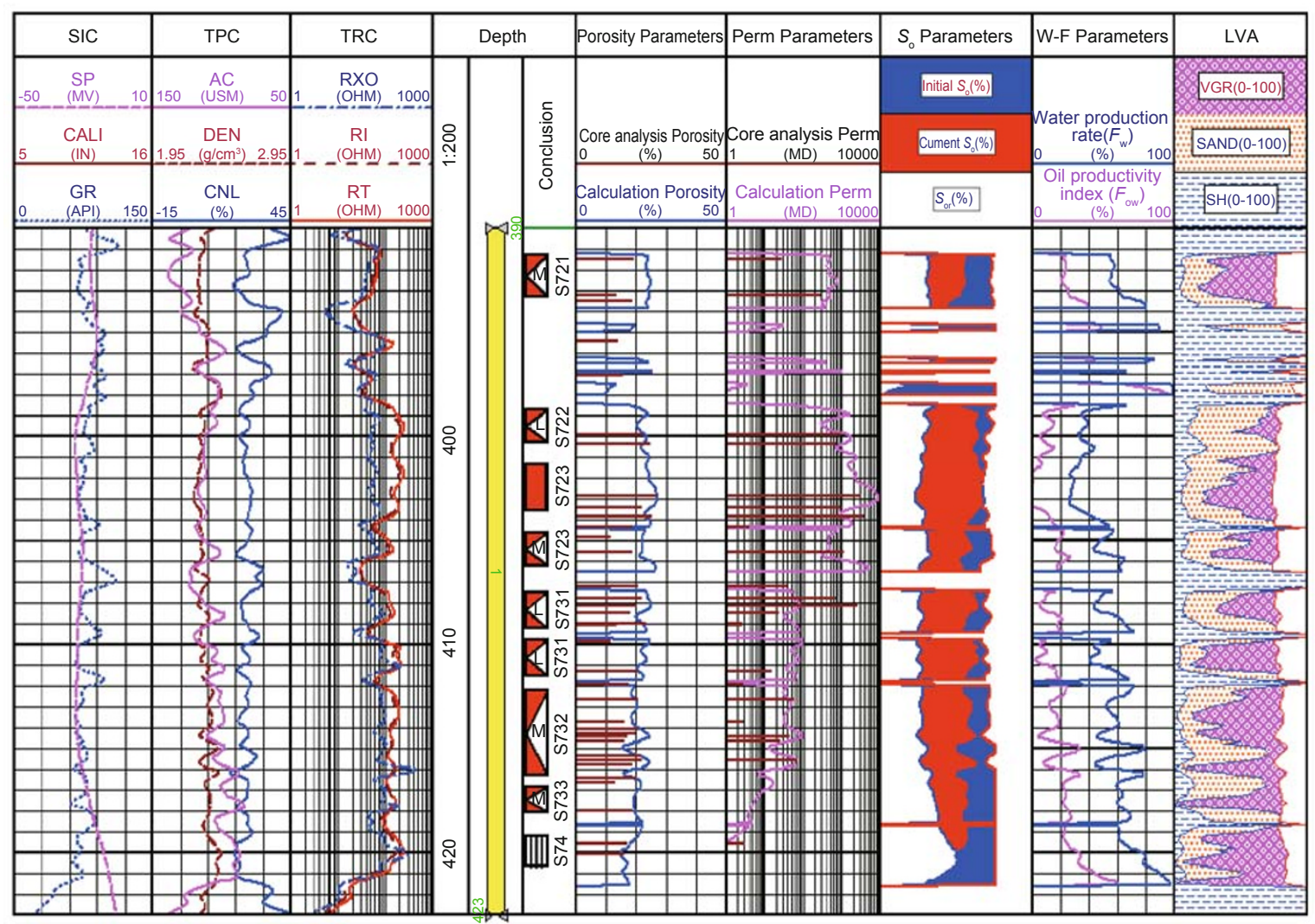

Notes: $S_{\text {or }}$ is the irreducible oil saturation of reservoir; VGR is the conglomerate content of formation.

Fig. 7 The results of quantitative interpretation of water-flooded layers for well J581

S74, S73, S72, S71 and S6 from bottom to top. S73 is an important production formation and it is divided into three sublayers S733, S732 and S731. For layer S733 the daily fluid production rate was $15.81 \mathrm{t}$ and the daily oil production was $4.38 \mathrm{t}$, so the water content was $72.3 \%$, while the quantitative interpretation result of layer S733 was mediumstrong flooded layer. For layer S732 the daily fluid production rate was $7.23 \mathrm{t}$ and the daily oil production was $3.99 \mathrm{t}$, so the water content was $44.8 \%$, while the quantitative interpretation result of S732 was medium flooded layer. For layer S731 the daily fluid production rate was $10.63 \mathrm{t}$ and the daily oil production was $7.69 \mathrm{t}$, so the water content was $27.7 \%$, while the quantitative interpretation result of S731 was weakly flooded layer. From the above analysis it can be seen that the interpretation conclusion and testing result are basically consistent and this method is good for quantitative evaluation of water-flooded layers for the conglomerate reservoir.
The water-flooded layers of more than 120 new wells in the Sixth District have been quantitatively interpreted in 2007-2008 by use of the quantitative evaluation method for water-flooded layers, and a total of 24 oil layers were avoided as strongly water-flooded and 284 perforated intervals were reasonably adjusted. The liquid production capacity was 839 t/d and oil production capacity was 286 $\mathrm{t} / \mathrm{d}$, so the water content was $65.9 \%$, after evaluation and adjustment for conglomerate reservoir of the Sixth District. The water content had decreased by $13.3 \%$ compared with $79.2 \%$ in 2006 . Finally, the use of oil productivity index and integration of various parameters can greatly improve the accuracy of quantitative interpretation of water-flooded layers for a conglomerate reservoir, and provide effective technical support for adjustment of well patterns and establishment of developmental programs in the second development stage of the conglomerate reservoir. 


\section{Conclusions}

1) The special sedimentary environment of a conglomerate reservoir leads to complex and changeable lithology and severe heterogeneity, it is difficult to establish models of lithology identification for the methods of traditional geophysics by use of various information of well logging curves, core testing data and geological information. But the data mining method with the ability of self-organization, selflearning, reasoning and thinking and high-precision nonlinear modeling can give a good solution to this problem. The "white-box" model of Decision Tree can clearly understand how to identify different lithologies according to different parameter combination and association rules. It gives a good guide for geophysics study.

2) Multiple linear regression of the water saturation avoids complex questions of calculating formation water resistivity and it is replaced by the continuous normalized values of the spontaneous potential curves, improving the accuracy of the oil saturation calculation.

3) The oil productivity index represents a dynamic waterflooded parameter from the beginning of water-flood to the current state of the reservoir. It eliminates the influence of conglomerate lithology by use of the relative value of oil saturation and avoids the shortcomings in quantitative evaluation of the water-flooded extent by using only the current oil saturation. The sensitivity of the oil productivity index is the highest and the calculated accuracy is the best among the three characteristics parameters of water-flood layers.

4) It can effectively evaluate water-flooded levels of a conglomerate reservoir in combination with the three characteristics parameters of water-flood layers and reservoir resistivity, the methods were applied to quantitative evaluation of water-flooded layers of the Kexia Group conglomerate reservoir of the Sixth District, and achieved very good application effects. It will provide technical support for avoiding perforation of high water-bearing layers and for adjustment of developmental program.

\section{Acknowledgements}

The authors would like to thank Qin Jianhua from the Research Institute of Petroleum Exploration and Development in Xinjiang Oilfield Company for their helpful discussion and suggestions.

\section{References}

Cheng $\mathrm{X}$ and Ren Z L. Cased-hole resistivity logging reservoir evaluation of petroleum feature changes. Journal of Northwest
University (Natural Science Edition). 2008. 38(3): 462-466 (in Chinese)

Fu Z H, Zhang Z Z, Li D C, et al. Analysis of sedimentary systems and hydrocarbon accumulation of Palaeogene, Chengbei Sag. Acta Sedimentologica Sinica. 2009. 27(1): 25-31 (in Chinese)

Fayyad U. Gregory Piatetsky-Shapiro, Padhraic Smyth. Knowledge Discovery and Data Mining: Towards a Unifying Framework. Portland. Oregon: AAAI Press. 1996: 82-88

He J X, Xia B, Wang Z X, et al. Hydrocarbon accumulation and exploratory orientation in the western marginal basin of the northern South China Sea. Acta Petrolei Sinica. 2006. 27(4): 8-14 (in Chinese)

Han J and Kamber M. Data Mining: Concepts and Techniques (The Morgan Kaufmann Series in Data Management Systems). Second Edition, Morgan Kaufmann, 2006

Jiang B X, Zhang P, Wang H, et al. Study of conglomerate reservoir water flooded zones in the Karamay oilfield. Gas Petroleum and Development, 2005, 28(3): 56-75 (in Chinese)

Ouyang J. Petroleum well logging interpretation and reservoir description. Beijing: Petroleum Industry Press. 1994. 220-229 (in Chinese)

Pan H P and Wang X. Research into computational methods of reservoir original oil saturation. Modern Geology. 2000. 14(4): 452-453 (in Chinese)

Sun J M, Wang K W, Li W, et al. Development and analysis of logging saturation interpretation models. Petroleum Exploration and Development. 2008. 35(1): 101-107 (in Chinese)

Shen J S, Su B Y, Wang Z R, et al. Analysis and contrast of the shaly sandstone conductivity model. Well Logging Technology. 2008. 32(5): 385-393 (in Chinese)

Shen H L, Kuang L C, Yang H, et al. Application of multiwell logging interpretation techniques to evaluation of water flooded zones in the Karamay conglomerate reservoir. Journal of the University of Petroleum, China (Edition of Natural Science). 1998. 22(2): 21-25 (in Chinese)

Tian M M. A summary of decision trees in data mining. Journal Changchun University. 2004. 14(6): 48-51 (in Chinese)

Wang Z Y and Zhao W Z. Study of the interaction of overpressure and hydrocarbon generation and the influence of overpressure upon hydrocarbon accumulations. Petroleum Exploration and Development. 2002. 29(4): 12-15 (in Chinese)

Worthington P F. Character of water saturation algorithms through dualsalinity desaturation. The Log Analyst. 1996. 37(1): 31-46

Witten I H and Frank E. Data Mining: Practical Machine Learning Tools and Techniques. Second Edition, Morgan Kaufmann. 2005

Yu H H. Application of well logging interpretation by capillary theorycapillary electrodynamics and variety porosity rock. Beijing: Petroleum Industry Press. 1995. 9-11 (in Chinese)

Zhang W C, Xiang S Q, Chen Y J, et al. "Facies-potential-transport" reservoir model and formation of reservoirs in fault basins. Acta Petrolei Sinica. 2008. 29(5): 680-693 (in Chinese)

Zhang Y G, Ma Z J, Wang G L, et al. The hydrocarbon reservoir mode of marine sedimentary rocks in south China. Acta Geologica Sinica. 2007. 81(2): 236-243 (in Chinese)

(Edited by Zhu Xiuqin) 